\title{
Online Primary Channel Selection for Dynamic Channel Bonding in High-Density WLANs
}

\author{
Sergio Barrachina-Muñoz, Francesc Wilhelmi, and Boris Bellalta
}

\begin{abstract}
In order to dynamically adapt the transmission bandwidth in wireless local area networks (WLANs), dynamic channel bonding (DCB) was introduced in IEEE 802.11n. It has been extended since then, and it is expected to be a key element in IEEE 802.11ax and future amendments such as IEEE 802.11be. While DCB is proven to be a compelling mechanism by itself, its performance is deeply tied to the primary channel selection, especially in high-density (HD) deployments, where multiple nodes contend for the spectrum. Traditionally, this primary channel selection relied on picking the most free one without any further consideration. In this paper, in contrast, we propose dynamic-wise (DyWi), a light-weight, decentralized, online primary channel selection algorithm for DCB that improves the expected WLAN throughput by considering not only the occupancy of the target primary channel but also the activity of the secondary channels. Even when assuming important delays due to primary channel switching, simulation results show a significant improvement both in terms of average delay and throughput.
\end{abstract}

Index Terms-Dynamic channel bonding, primary channel, high-density WLAN, online

\section{INTRODUCTION}

$\mathbf{M}$ ODERN applications like augmented reality, virtual reality, or real-time $8 \mathrm{~K}$ video are pushing next-generation (nextGen) wireless local area networks (WLANs) to support ever-increasing demands on performance. In addition, the complexity of nextGen high-density (HD) deployments, where numerous wireless devices will contend for accessing the medium, hinders even more the task of achieving high throughput and low latency.

In order to improve the performance of nextGen WLANs, we focus in this work on spectrum management techniques. In particular, two well-known techniques have been widely studied in this regard: channel allocation (CA) and dynamic channel bonding (DCB). CA is the method to assign portions of the spectrum (or channels) to one or multiple WLANs. In contrast, DCB is a technique whereby two or more channels are bonded according to their instant occupancy, enabling wider bandwidths per transmission, and thus potentially reaching higher data rates.

Although much has been understood from the works on CA and DCB in the literature, little has been assessed with respect to combining CA with DCB altogether in WLANs, particularly

All the authors are with the Wireless Networking research group at Universitat Pompeu Fabra, Barcelona, Spain (e-mail: \{sergio.barrachina, francisco.wilhelmi, boris.bellalta $@$ upf.edu). This work has been partially supported by the Spanish Ministry of Economy and Competitiveness under the Maria de Maeztu Units of Excellence Programme (MDM-2015-0502), by a Gift from the Cisco University Research Program (CG\#890107, Towards Deterministic Channel Access in High-Density WLANs) Fund, a corporate advised fund of Silicon Valley Community Foundation, and by PGC2018 099959-B-I00 (MCIU/AEI/FEDER,UE). The work done by S. BarrachinaMuñoz is supported by a FI grant from the Generalitat de Catalunya. for high-density (HD) deployments. Nevertheless, while DCB has shown a tremendous potential to outperform traditional single-channel (SC) [1]-[3], its performance is severely tied to the CA. Especially, the primary channel selection is critical since it runs the backoff procedure of the carrier sense multiple access with collision avoidance (CSMA/CA) protocol. Still, always selecting the least occupied channel as the primary is no longer appropriate for DCB since potential bonds with adjacent channels should be also considered.

As for works combining CA and DCB for WLANs, we find a distributed algorithm for jointly allocating channel center frequencies and bandwidths [4], or a centralized approach for maximizing the network fairness [5]. In addition, a heuristicbased algorithm for primary channel selection based on the bonding direction likelihoods was recently presented in [6]. However, such likelihoods are estimated by assuming a known number of users in each channel, which is normally not feasible in real deployments. It is worth noticing that the aforementioned works consider fully-backlogged traffic, thus missing insights on more realistic patterns with different traffic needs. Finally, an uncertain traffic CA approach was presented in [7]. Still, a centralized controller in the backend is required.

In this paper, we formulate dynamic-wise (DyWi), a decentralized, lightweight algorithm that leverages information about the sensed spectrum occupancy in the whole allocated bandwidth of a node (i.e., primary and secondary channels). Based on such occupancy, the primary channel is selected in an online manner with the aim of maximizing the expected throughput. It does so by considering not only the activity of the target primary channel but also the potential bonds that could be established with its adjacent channels. DyWi is adaptive in the sense that a new primary channel is only adopted when the WLAN performance is below a given satisfaction threshold. Besides, since DyWi relies just on local information, neither neighbor messaging nor a central controller is required. This property makes DyWi suitable to be implemented in off-the-shelf access points (APs), thus avoiding costly interWLAN collaboration.

Simulations of DyWi in IEEE 802.11ax HD deployments show important improvements with respect to traditional primary channel selection, even when considering substantial delays due to channel switching.

\section{PRimary CHANNEL SELECTION FOR DCB}

\section{A. Dynamic channel bonding}

DCB was first introduced in IEEE 802.11n (2009), where two contiguous $20-\mathrm{MHz}$ channels could be bonded to form a single 40-MHz channel. Then, IEEE 802.11ac (2013) extended 


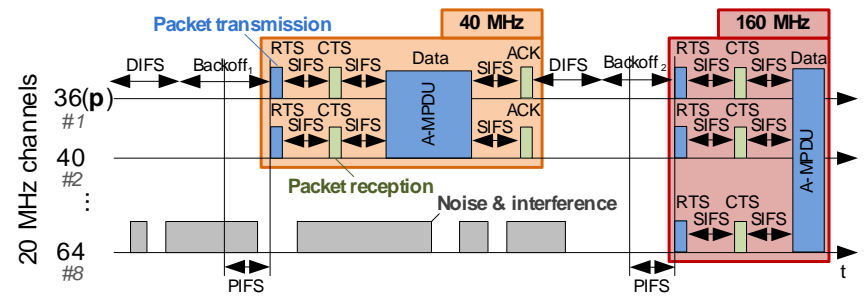

Fig. 1: CSMA/CA operation of a node operating under DCB in the IEEE 802.11ac/ax channelization scheme for the UNII-1 and UNII-2 bands. The numbers preceded by a pound symbol (\#) represent a simpler channel indexation.

the DCB capability to bond up to $820-\mathrm{MHz}$ channels, reaching a maximum of $160-\mathrm{MHz}$ transmission bandwidth. While IEEE 802.11ax (2019) keeps such a limit, future amendments like EXtreme throughput (i.e., IEEE $802.11 \mathrm{be}$ ) aim to support up to $320-\mathrm{MHz}$ transmissions. Figure 1 shows the operation timeline of a node implementing DCB with primary channel $p=1$. Note that the bandwidth selection is decided according to the occupancy of the secondary channels during the PCF Interframe Space (PIFS) previous to the backoff termination. Accordingly, transmissions of 40 and $160 \mathrm{MHz}$ are performed after the expiration of the first and second backoff in the example, respectively.

\section{B. Online selection of the primary channel}

Let an AP belonging to WLAN $w$ operate under DCB and have allocated the full available bandwidth according to a channelization scheme $C$. As an example, the channelization (or allowed transmission channels) in Fig. 1 is $C=$ $\{\{1\},\{2\}, \ldots,\{8\},\{1,2\}, \ldots,\{7,8\},\{1,2,3,4\},\{5,6,7,8\},\{1, \ldots, 8\}\}$, where $C_{20-\mathrm{MHz}}=\{\{1\},\{2\}, \ldots,\{8\}\} \subset C$ is the set of $20-\mathrm{MHz}$ (basic or non-bonded) channels in $C$. Then, since the primary channel must be $20 \mathrm{-MHz}, p \in C_{20-\mathrm{MHz}}$. Note that throughout the rest of the paper, we use $b=20 \mathrm{MHz}$ to denote the bandwidth of a basic channel.

For the sake of identifying a convenient primary channel in an online manner, we rely on an iterative algorithm. In essence, the AP of $w$ periodically makes a decision about the primary channel selection, where each decision instant represents the beginning of an iteration. Specifically, at the beginning of a given iteration $t$, the $\mathrm{AP}$ of $w$ measures the throughput $s_{w, t-1}$ achieved during the last iteration $t-1$ and acts according to a satisfaction condition. ${ }^{1}$ Namely, the primary channel remains the same if $w$ is satisfied because a sufficient share of the generated traffic has been successfully sent during the last iteration, ${ }^{2}$ i.e., $s_{w, t-1} \geq \eta \ell_{w, t-1}$, where $\eta$ is the satisfaction ratio and $\ell_{w, t-1}$ is the actual generated traffic load in that iteration. Otherwise, $w$ will switch to a new primary channel at the cost of remaining inactive during a period $\delta$, required to announce the new primary channel to the associated stations

\footnotetext{
${ }^{1}$ In this work, we focus on the successful downlink traffic as the main performance metric. However, the algorithm can be easily extended to consider other parameters such as latency.

${ }^{2}$ We rely just on data from the last iteration for lowering memory demands and enabling fast adaptability in dynamic environments.
}

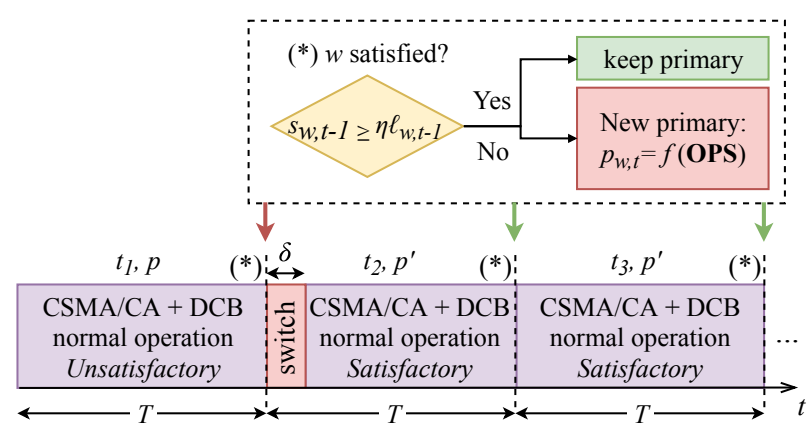

Fig. 2: Example of the satisfaction-based online primary channel selection. Since $w$ is not satisfied at the end of iteration $t_{1}$, the primary channel is switched from $p_{w, 1}=p$ to $p_{w, 2}=p^{\prime}$, determined by a given OPS rule. In contrast, with the new primary, $w$ gets satisfied in $t_{2}$ and keeps $p^{\prime}$ in $t_{3}$.

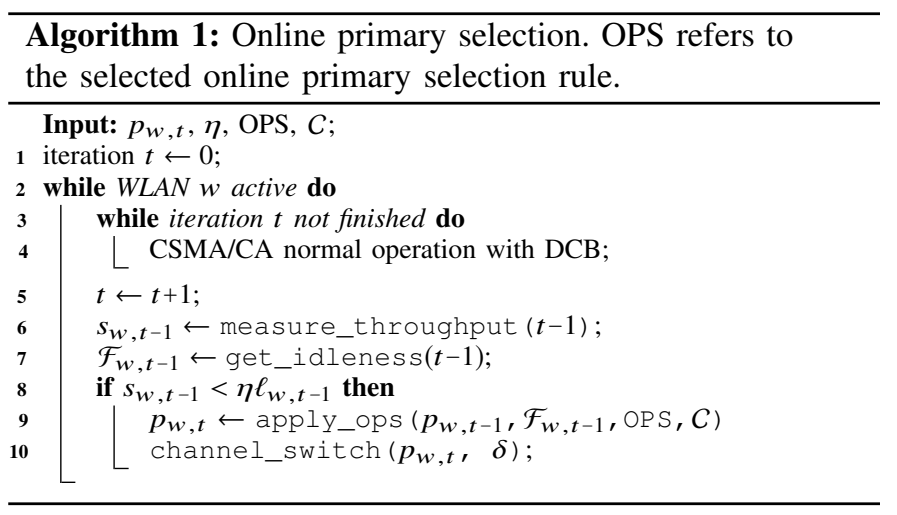

(STAs), and to apply the new channel configuration. Note that the way the new primary channel is selected depends on the online primary selection (OPS) rule. The temporal evolution of the general procedure is displayed in Fig. 2 through an illustrative example.

Algorithm 1 shows the pseudocode of the online primary channel selection for a generic OPS rule. Note that any OPS rule relies on the gathered data about the bandwidth occupancy in the last iteration. Formally, the empirical probability that a subset of $n$ channels in channelization $C$ was free (or idle) during the last iteration $t-1$ given a primary $p_{w, t}$ is given by

$$
\mathcal{F}_{w, t-1}\left(C, p_{w, t}, n\right)=\mathbb{E}_{t-1}\left[P_{\mathrm{rx}}(c)<\mathrm{CCA}, \forall c \in C\left(p_{w, t}, n\right)\right],
$$

where $P_{\mathrm{rx}}(c)$ is the power received at basic channel $c$, CCA is the clear channel assessment (CCA) threshold, and $C\left(p_{w, t}, n\right)$ is the set of basic channels used in the transmission, which is mandated by the channelization scheme $C$. For instance, following the IEEE 802.11ac/ax channelization, for primary $p=6$ and $n=2$, the corresponding $40-\mathrm{MHz}$ bonded channel is $C(6,2)=\{5,6\}$ (channels \#52 and \#56 in the standard). With slight abuse of notation, $\mathbb{E}_{t-1}$ represents the expected value function at iteration $t-1$.

Three OPS rules are evaluated in this work: dynamicrandom (DR), dynamic-free (DF), and dynamic-wise (DiWy or DW). In the event of an unsatisfactory iteration, DR selects a new primary channel uniformly at random, DF picks the one 


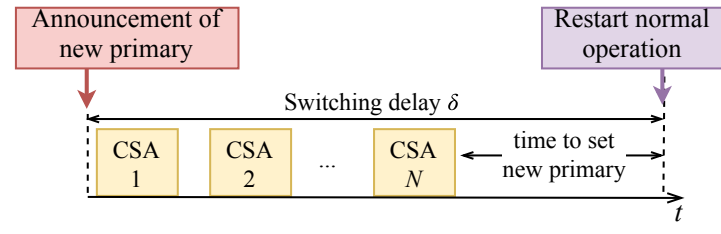

Fig. 3: Channel switch procedure.

found most free during the last iteration, and DyWi selects it based on the forecast throughput given the probability of bonding in every possible bandwidth. Such probabilities are estimated by periodically measuring the energy in all the secondary channels as done during the PIFS period. Apart from DR and DF, we also consider as baseline the traditional fix primary (FP) allocation, which does not change the primary channel under any circumstances, so it is not referred in Algorithm 1.

Regarding the channel switch operation, the $802.11 \mathrm{~h}$ standard (later included in amendments like 802.11ax) states that the AP may notify the associated STAs through Channel Switch Announcement (CSA) frames [8]. As depicted in Fig. 3, the channel switch is scheduled so that all the STAs in the WLAN have the opportunity to receive at least one CSA element before the actual switch. Specifically, the number of remaining frames to be broadcast until switching to the new primary channel is known as CSA count value. Accordingly, the switching delay $\delta$ is the sum of the time it takes to broadcast the CSA frames and the time left to STAs to adapt to the new primary channel.

\section{Dynamic-wise (DyWi) primary channel selection}

A main question arises when considering DyWi regarding the way the primary channel should be selected. Assume a scenario where a WLAN $w$ is allocated $80 \mathrm{MHz}$ accounting from channel 1 to 4 . At the end of iteration $t-1, w$ is unsatisfied and changes its primary from $p_{w, t-1}=2$ to $p_{w, t}$. Assume also that the probabilities of finding free each of its allocated $20-\mathrm{MHz}$ channels in iteration $t-1$ was $\boldsymbol{\pi}_{w, t-1}=[0.93,0.38,0.85,0.85]$, where $\pi_{w, t-1}[c]$ is the probability of finding the basic channel $c$ free. Then, two main options may be contemplated as best choice for selecting $p_{w, t}$ to maximize the throughput $s_{w, t}$ of the upcoming iteration: $i$ ) to pick the primary with highest probability to be free (i.e., $p_{w, t}=1$ in this case), or $\left.i i\right)$ to pick the primary providing the highest potential average data rate considering both its probability to be free, as well as the probability of the channels nearby (e.g., $p_{w, t} \in\{3,4\}$ ). Notice that there is not an accurate choice to provide beforehand since multiple parameters apart from $\pi_{w, t-1}$ will impact the performance. For example, dynamic network activity affects to the signal-to-interference-plus-noise ratio (SINR), modulation coding scheme (MCS), or packet error rate.

We tackle this point at issue by proposing a lightweight maximization problem for the forecast data rate of WLAN $w$ at iteration $t$. The idea behind this approach is that maximizing the successful data rate should lead to maximizing the throughput as well. Thus, in spite of being a sub-optimal formulation for maximizing the throughput, ${ }^{3}$ selecting the primary channel according to the forecast average data rate is a convenient heuristic, as shown later. In particular, the average data rate is estimated by the probability of transmitting at each possible bandwidth. Then, we can formulate the problem as

$$
\begin{aligned}
& \underset{p_{w, t} \neq p_{w, t-1}}{\operatorname{argmax}} \hat{r}_{w, t}\left(p_{w, t}\right), \text { with } \\
& \hat{r}_{w, t}\left(p_{w, t}\right)=\sum_{n \in \mathcal{N}} \mathbb{P}_{w, t}\left(\mathcal{F}_{w, t-1}, p_{w, t}, n\right) r_{w}(n),
\end{aligned}
$$

where $\hat{r}_{w, t}\left(p_{w, t}\right)$ is the forecast average data rate by WLAN $w$ at iteration $t$ for new primary $p_{w, t}$, and $n$ is the number of bonded channels, which should be permitted by the channelization scheme. For instance, in the IEEE 802.11ax amendment, $n \in \mathcal{N}=\{1,2,4,8\}$, for 20 to $160 \mathrm{MHz}$ bandwidths. $\mathbb{P}_{w, t}\left(\mathcal{F}_{w, t-1}, p_{w, t}, n\right)$ is the probability that $w$ transmits in $n$ contiguous channels in the starting iteration given $p_{w, t}$ is selected, and $r_{w}(n)$ is the data rate given the bandwidth $n b$. Note that $r$ also depends on the MCS index, which will vary according to the signal-to-noise ratio (SNR) at the STA.

In order to estimate the probability of transmitting in each possible combination of channels, we rely on the empirical probability $\mathcal{F}_{w, t-1}\left(C, p_{w, t}, n\right)$, which was updated during the CSMA/CA operation in iteration $t-1$. Since DCB is implemented, the largest available bandwidth is always picked per transmission. Hence, the probability of transmitting in a certain bandwidth is contingent on the probability of transmitting in higher bandwidths. Specifically,

$$
\begin{array}{r}
\mathbb{P}_{w, t}\left(\mathcal{F}_{w, t-1}, p_{w, t}, n\right)=\mathcal{F}_{w, t-1}\left(C, p_{w, t}, n\right) \\
-\sum_{n^{\prime} \in\left\{\mathcal{N} \mid n^{\prime}>n\right\}} \mathbb{P}_{w, t}\left(\mathcal{F}_{w, t-1}, p_{w, t}, n^{\prime}\right),
\end{array}
$$

where the probability of transmitting in wider bandwidths is subtracted due to the constraint $n^{\prime} \in\left\{\mathcal{N} \mid n^{\prime}>n\right\}$. For instance, for bands UNII-1 and UNII-2 altogether (i.e., $\left.C_{20-\mathrm{MHz}}=\{1, \ldots, 8\}\right)$, we define $\mathbb{P}(\mathcal{F}, p, 8)=\mathcal{F}(C, p, 8)$ for $n=8$ (i.e., $160-\mathrm{MHz})$. Similarly, on the other end, $\mathbb{P}(\mathcal{F}, p, 1)=$ $\mathcal{F}(C, p, 1)-\mathbb{P}(\mathcal{F}, p, 2)-\mathbb{P}(\mathcal{F}, p, 4)-\mathbb{P}(\mathcal{F}, p, 8)$ for $n=1$ (i.e., 20-MHz).

As for the complexity of the presented OPS rules, note that they are all computational lightweight; especially DR since it does not keep track of any data. Despite DF's complexity increases with the number of basic channels, $\mathcal{F}_{\mathrm{DF}}\left(\left|C_{20-\mathrm{MHz}}\right|\right)$, its complexity remains also low. DyWi's complexity, however, is bounded by $\mathcal{F}_{\mathrm{DW}}\left(\left|C_{20-\mathrm{MHz}}\right|\left(\log _{2}\left|C_{20-\mathrm{MHz}}\right|+1\right)^{2}\right)$ for the IEEE 802.11ax channelization. Nonetheless, DyWi is completely tractable in operation time by off-the-shelf network cards since the number of possible bonds in the 5-GHz band is still small.

\section{SYSTEM MODEL}

For evaluating the performance of the OPS rules presented in Section II, we simulate IEEE 802.11ax HD deployments using the Komondor [9] wireless network simulator v1.2.1c. For simplicity, we consider negligible propagation delay, downlink

\footnotetext{
${ }^{3}$ One should consider different complex parameters such as the buffer status and environment dynamics to define an optimal formulation.
} 
TABLE I: Evaluation setup.

\begin{tabular}{ccc}
\hline Param. & Description & Value \\
\hline$f_{\mathrm{c}}$ & Central frequency & $5.25 \mathrm{GHz}$ \\
$b$ & Basic channel bandwidth & $20 \mathrm{MHz}$ \\
$L_{\mathrm{d}}$ & Data packet size & $12000 \mathrm{bits}$ \\
$N_{\mathrm{b}}$ & Buffer capacity & 150 packets \\
$N_{\mathrm{a}}$ & Max. no. of aggregated packets in a frame & 64 \\
$\mathrm{CW}_{\min }$ & Min. contention window & 16 \\
$m$ & No. of backoff stages & 5 \\
$\mathrm{MCS}$ & IEEE 802.11ax MCS index & $0-11$ \\
$\eta$ & MCS's packet error rate & 0.1 \\
$\mathrm{CCA}$ & CCA threshold & $-82 \mathrm{dBm}$ \\
$P_{\mathrm{tx}}$ & Transmission power & $15 \mathrm{dBm}$ \\
$G_{\mathrm{tx}}$ & Transmitting gain & $0 \mathrm{~dB}$ \\
$G_{\mathrm{rx}}$ & Reception gain & $0 \mathrm{~dB}$ \\
$\mathrm{PL}(d)$ & TMB indoor path loss for 11ax & see $[11]$ \\
$\mathrm{CE}$ & Capture effect threshold & $20 \mathrm{~dB}$ \\
$N$ & Background noise level & $-95 \mathrm{dBm}$ \\
\hline$C$ & Channelization for UNII-1 \& UNII-2 & $36(1)-64(8)$ \\
$\left|C_{20-\mathrm{MHz}}\right|$ & No. of 20-MHz channels in the system & 8 \\
$\mathcal{N}$ & Allowed no. of bonded channels & $\{1,2,4,8\}$ \\
$\mathrm{OPS}$ & Online primary selection rule & DR, DF, DW \\
$T$ & Iteration duration & $1 \mathrm{~s}$ \\
$T_{\mathrm{obs}}$ & Simulation duration & $25 \mathrm{~s}$ \\
$\eta$ & Satisfaction ratio & 0.9 \\
$\delta$ & Switching delay & $0,100 \mathrm{~ms}$ \\
\hline
\end{tabular}

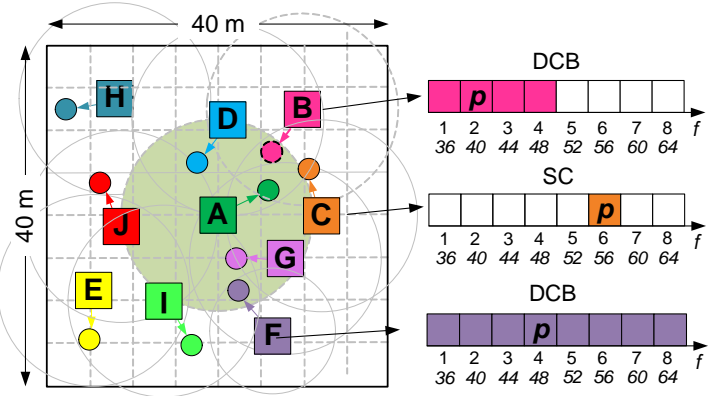

Fig. 4: Deployment with WLAN A in the center. The IEEE 802.11ac/ax 20-MHz channel indexation is displayed in italic. WLANs may implement SC or DCB in different allocated bandwidths.

traffic, and WLANs composed by one AP and one STA. The packet arrival process at each AP follows a Poisson process, generating packets every $t_{n} \sim \operatorname{Exponential}\left(L_{\mathrm{d}} / \bar{\ell}\right)$, where $L_{\mathrm{d}} / \bar{\ell}$ is the average packet arrival rate given a fixed packet length $L_{\mathrm{d}}$ and average arrival bit rate $\bar{\ell}[10]$. As for the packet reception model, we consider that a packet is lost if the SINR perceived at the receiver is below the capture effect (CE) threshold. Note that transmitted power is spread over the channels used in the transmission bandwidth. We also consider the same CCA $(-82 \mathrm{dBm})$ both in primary and secondary channels to make channel access more restrictive, as proposed in [1].

Regarding the simulation deployments, we contemplate dense $40 \times 40 \mathrm{~m}^{2}$ scenarios like the one shown in Fig. 4. One WLAN (A) remains always located at the center in every scenario, and other 9 WLANs are spread uniformly at random in the area. The only condition is that any pair of APs must be separated at least $d_{\mathrm{AP}-\mathrm{AP}}^{\min }=10 \mathrm{~m}$. The STA of each WLAN is located also uniformly at random at a distance $d_{\mathrm{AP}-\mathrm{STA}} \in\left[d_{\mathrm{AP}-\mathrm{STA}}^{\min }, d_{\mathrm{AP}-\mathrm{STA}}^{\max }\right]=[1,5] \mathrm{m}$ from the AP. Note that

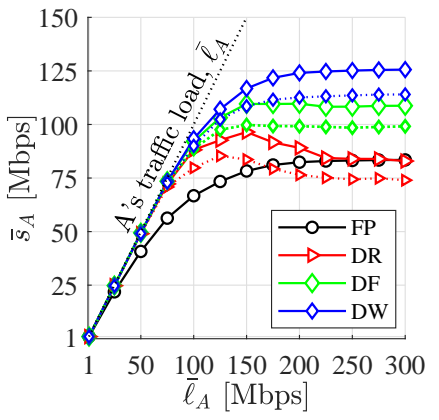

(a) Average throughput. (b) Prob. of desired throughput.

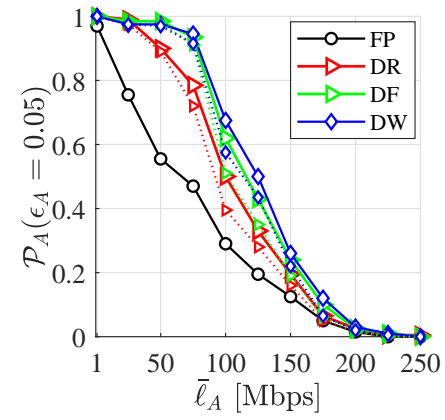

Fig. 5: Performance metrics of WLAN A for the different primary selection rules. Continuous lines assume no switching cost while dashed lines correspond to $\delta=100 \mathrm{~ms}$.

WLANs are not required to be within the carrier sense range of the others, i.e., the simulations capture spatial distribution effects.

Regarding the CA, all the WLANs are set with a random primary channel in the eight basic channels of the UNII$1 \& 2$ sub-bands (i.e., $p_{\mathrm{w}} \sim U[1,8], \forall w$ ). The set of allocated basic channels is assigned uniformly at random as well. That is, the number of allowed basic channels for transmitting in $w$ is $\left|C_{w}\right| \sim U\{1,2,4,8\}, \forall w$, except for WLAN A, which is allocated the whole bandwidth in all the scenarios (i.e., $\left.C_{\mathrm{A}}=\{1, \ldots, 8\}\right)$. While A implements DCB, the rest of WLANs implement SC or DCB with same probability $1 / 2$ ).

We generate $N_{\mathrm{D}}=200$ random deployments following the aforementioned conditions and evaluate $N_{\ell}=17$ values of A's average traffic load $\bar{\ell}_{A}$ ranging from 1 to $400 \mathrm{Mbps}$. The rest of WLANs are set with random average traffic load inside this range, i.e., $\bar{\ell}_{w} \sim U[1,400]$ Mbps. In addition to traditional FP, we consider the $N_{\mathrm{P}}=3$ OPS schemes proposed in Section II (i.e., DR, DF, DW). For the latter ones, two switching delay costs $\delta \in\{0,100\}$ ms are assessed. ${ }^{4}$ Consequently, we simulate $N_{\mathrm{D}} \times N_{\ell} \times\left(1+2 N_{\mathrm{P}}\right)=23,800$ scenarios. The simulation time of each scenario is $T_{\mathrm{obs}}=25$ seconds. As for the configuration of the online algorithms, we set the iteration time $T=1 \mathrm{~s}$ and satisfaction ratio $\eta=0.9$. Note that we consider a value of $\eta$ smaller than 1 to provide stability to the algorithm.

\section{Performance Evaluation}

Figs. 5 and 6 collect key performance metrics of WLAN A in the proposed scenario for different traffic loads. Namely, Fig. 5a shows the average throughput $\bar{s}_{\mathrm{A}}$ computed as the number of bits successfully transmitted (acknowledged) divided by the simulation time $T_{\mathrm{obs}}$. The probability $\mathcal{P}_{\mathrm{A}}$ of successfully transmitting sufficient traffic is plotted at Fig. 5b. Such a probability is computed as the portion of scenarios accomplishing $\bar{s}_{\mathrm{A}} \geq\left(1-\epsilon_{s}\right) \bar{\ell}_{\mathrm{A}}$, where $\epsilon_{s}=0.05$ is set to deal with the non-deterministic traffic generation. Finally, Fig. 6 shows the average packet delay $\bar{d}_{\mathrm{A}}$.

\footnotetext{
${ }^{4}$ The number and time between CSA frames are configurable (and dependant on the AP manufacturer). In this work, we consider a practical value $\delta=100 \mathrm{~ms}$ that allows stressing the performance of the evaluated algorithms.
} 


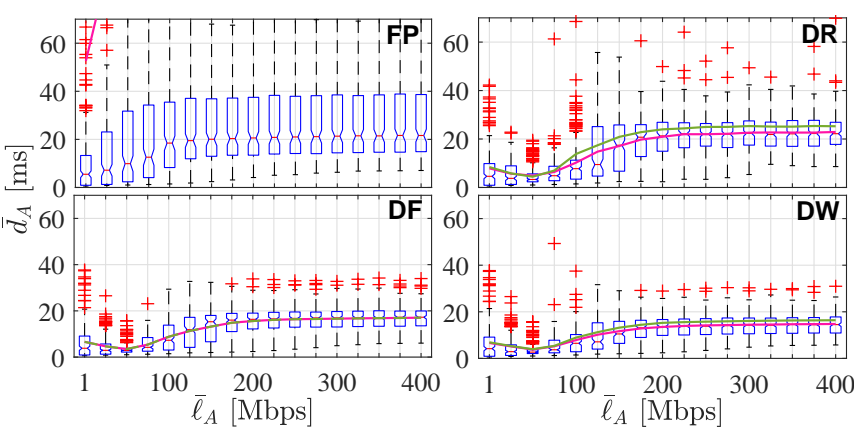

Fig. 6: Distribution of the average delay of A for $\delta=0 \mathrm{~ms}$. The mean value for the different loads is represented through the purple and green lines for $\delta=0$ and $\delta=100 \mathrm{~ms}$, respectively.

As shown by the different metrics, DyWi clearly outperforms FP and DR both in terms of throughput and delay. In fact, even when considering a huge adaptation cost delay $\delta=100 \mathrm{~ms}$ (i.e., an important $10 \%$ penalty with respect to the iteration duration $T$ ), $\mathcal{P}_{A}$ is prominently increased for moderate and medium loads. While DR may be counterproductive for high loads (see Fig. 5a), both DF and DyWi keep a constant performance after saturating. Such a throughput reduction for DR is caused by the fact that the larger $\bar{\ell}_{A}$, the harder to remain satisfied, which leads to more frequent channel switching. Then, critically for DR, the random selection of the primary leads to picking each channel with the same probability. Accordingly, the average throughput converges to FP's because, on average (for all the scenarios), the primary channels are equiprobable selected whenever the satisfaction condition is not accomplished.

As for the average delay, even though we can see by the outliers in Fig. 6 that adopting (2) does not guarantee optimal performance for every scenario, DyWi outperforms the rest of selection rules in most of the cases. A phenomenon worth noticing is the reduction of the average delay from low to moderate traffic loads for online selection algorithms. The reason lies in the buffer dynamics. Specifically, during the normal operation of an unsatisfactory iteration, while for low traffic loads, the number of aggregated packets per frame is pretty low, it is much greater for higher loads. Accordingly, since the buffer tends to remain unsaturated for low loads, during the new configuration setup after an unsatisfactory iteration, the buffer is normally filled up. As a result, the first frame of the next iteration - usually containing many aggregated data packets- affects much more to the average delay for low traffic loads than for high loads.

In order to assess the temporal evolution of the different algorithms, Fig. 7 plots the cumulative distribution function (CDF) of the number of iterations $k$ required to reach a satisfactory primary channel for moderate, medium and high traffic loads. As expected, the lower the load, the higher the value of $\operatorname{CDF}(k)$ for any $k$. Note that there are few unusual scenarios where the CDF varies for FP. Those are the cases where the load is almost adequate from the very beginning and the stochastic nature of the traffic generation makes the throughput to vary around $\eta \bar{\ell}_{A}$ as the simulation progresses.

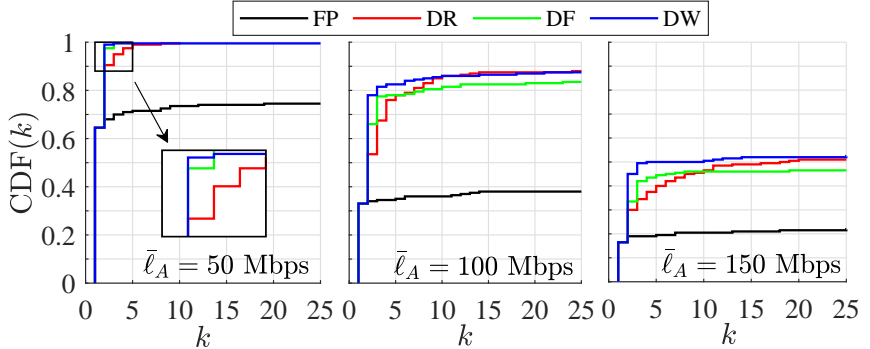

Fig. 7: Cumulative distribution function of the number of iterations $k$ required to reach satisfaction.

It is also observed that the highest value of the CDF is normally provided by DyWi, which wisely adapts to the medium. However, DR may be even better for intricate scenarios (high $k$ ) and medium/high loads. This suggests that for such difficult scenarios, relying on (2) may not be optimal due to the unexpected and harming interactions generated at the moment of changing the primary. Nevertheless, even though these unusual cases leave room for further improvement, DyWi is an effective solution that may be adopted in off-the-self WLANs due to its light-weight complexity and direct improvement.

\section{CONCLUSIONS}

In this work, we have formulated DyWi, a lightweight and decentralized online primary channel selection algorithm for WLANs. DyWi aims at maximizing the throughput by iteratively estimating the occupancy of the primary and secondary channels, thus boosting potential bonds. Results show significant improvements with respect to traditional allocation, even under high switching adaptation costs.

\section{REFERENCES}

[1] M. Park, "IEEE 802.11 ac: Dynamic bandwidth channel access," in Communications (ICC). IEEE, 2011, pp. 1-5.

[2] L. Deek, E. Garcia-Villegas, E. Belding, S.-J. Lee, and K. Almeroth, "Intelligent channel bonding in 802.11n WLANs," IEEE Transactions on Mobile Computing, vol. 13, no. 6, pp. 1242-1255, 2013.

[3] S. Barrachina-Muñoz, F. Wilhelmi, and B. Bellalta, "Dynamic channel bonding in spatially distributed high-density WLANs," (In press) IEEE Transactions on Mobile Computing, 2019.

[4] J. Herzen, R. Merz, and P. Thiran, "Distributed spectrum assignment for home WLANs," in INFOCOM. IEEE, 2013, pp. 1573-1581.

[5] B. Bellalta, A. Checco, A. Zocca, and J. Barcelo, "On the interactions between multiple overlapping WLANs using channel bonding," IEEE Transactions on Vehicular Technology, vol. 65, no. 2, pp. 796-812, 2016.

[6] S. Khairy, M. Han, L. X. Cai, Y. Cheng, and Z. Han, "A Renewal Theory based Analytical Model for Multi-channel Random Access in IEEE 802.11 ac/ax," IEEE Transactions on Mobile Computing, 2018.

[7] A. Nabil, M. J. Abdel-Rahman, and A. B. MacKenzie, "Adaptive channel bonding in wireless LANs under demand uncertainty," in Personal, Indoor, and Mobile Radio Communications (PIMRC). IEEE, 2017, pp. 1-7.

[8] IEEE, "Spectrum and transmit power management extensions in the 5 ghz band in europe," IEEE Std 802.11 h-2003, pp. 1-75, Oct 2003.

[9] S. Barrachina-Muñoz, F. Wilhelmi, I. Selinis, and B. Bellalta, "Komondor: a wireless network simulator for next-generation high-density WLANs," Wireless Days (WD), 2019.

[10] S. Barrachina-Muñoz, F. Wilhelmi, and B. Bellalta, "To overlap or not to overlap: enabling channel bonding in high-density WLANs," Computer Networks, vol. 152, pp. $40-53,2019$.

[11] T. Adame, M. Carrascosa, and B. Bellalta, "The TMB path loss model for $5 \mathrm{GHz}$ indoor WiFi scenarios: On the empirical relationship between RSSI, MCS, and spatial streams," Wireless Days (WD), 2019. 